\title{
NUMERICAL NORMAL FORMS FOR CODIM 2 BIFURCATIONS OF FIXED POINTS WITH AT MOST TWO CRITICAL EIGENVALUES*
}

\author{
YU. A. KUZNETSOV ${ }^{\dagger}$ AND H. G. E. MEIJER ${ }^{\ddagger}$
}

\begin{abstract}
In this paper we derive explicit formulas for the normal form coefficients to verify the nondegeneracy of eight codimension two bifurcations of fixed points with one or two critical eigenvalues. These include all strong resonances, as well as the degenerate flip and Neimark-Sacker bifurcations. Applying our results to $n$-dimensional maps, one avoids the computation of the center manifold, but one can directly evaluate the critical normal form coefficients in the original basis. The formulas remain valid also for $n=2$ and allow one to avoid the transformation of the linear part of the map into Jordan form.

The developed techniques are tested on two examples: (1) a three-dimensional map appearing in adaptive control; (2) a periodically forced epidemic model. We compute numerically the critical normal form coefficients for several codim 2 bifurcations occurring in these models. To compute the required derivatives of the Poincaré map for the epidemic model, the automatic differentiation package ADOL-C is used.
\end{abstract}

Key words. bifurcations of fixed points, normal forms, automatic differentiation

AMS subject classifications. 34C20, 37G05, 65P30

DOI. $10.1137 / 030601508$

1. Introduction. The study of a family of smooth discrete-time dynamical systems

$$
x \mapsto f(x, \alpha), \quad x \in \mathbb{R}^{n}, \alpha \in \mathbb{R}^{m},
$$

usually starts with the analysis of fixed points. Controlling one parameter, one encounters codimension 1 bifurcations of fixed points, i.e., critical parameter values where the stability of a fixed point changes. Continuation of such a point with respect to a second control parameter leads, generically, to a bifurcation curve. Two codim 1 bifurcation curves may intersect transversally or tangentially, depending on the nature of the bifurcations. Generically, at such parameter values a so-called codim 2 bifurcation occurs.

Two main issues are involved here. First, it is desirable to know what one can expect near a generic codim 2 point, i.e., to have canonical two-parameter local bifurcation diagrams for a given codim 2 bifurcation. Such diagrams have been derived for most of the codim 2 bifurcations of fixed points [14, 17]. Technical tools here are the center manifold reduction and the transformation to a normal form. Even though in many cases the bifurcation diagrams are in principle incomplete due to global phenomena, such as homoclinic or heteroclinic tangencies, many essential features of these diagrams are determined by the critical normal form coefficients. The second issue is how to apply these theoretical results to concrete discrete-time dynamical systems. This requires efficient algorithms for the computation of the critical normal

${ }^{*}$ Received by the editors October 29, 2003; accepted for publication (in revised form) September 2, 2004; published electronically May 20, 2005.

http://www.siam.org/journals/sisc/26-6/60150.html

${ }^{\dagger}$ Mathematisch Instituut, Universiteit Utrecht, P.O. Box 80010, 3508 TA Utrecht, The Netherlands; and Institute of Mathematical Problems of Biology, Russian Academy of Sciences, Pushchino, Moscow Region, 142290, Russia (kuznet@math.uu.nl).

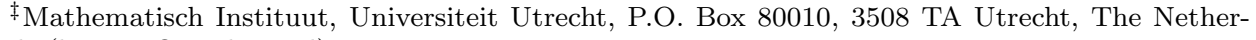
lands (hmeijer@math.uu.nl). 
form coefficients on the center manifold in terms of the original $n$-dimensional map (1.1). Our paper is devoted to the latter problem for a class of codim 2 bifurcations of fixed points with at most two critical eigenvalues. Once constructed, such algorithms provide useful initial guesses where to search for global phenomena in applications. Let us remark that the genericity of a bifurcation in a given system also requires its nondegeneracy with respect to actual control parameters, which should unfold the singularity transversally. We briefly discuss this problem at the end of the paper.

There are three codim 1 bifurcations of fixed points of maps, namely, fold, perioddoubling, and Neimark-Sacker. Encountering such a bifurcation one may use the formulas for the normal form coefficients derived via the central manifold reduction in [14, pp. 181-186] to check its nondegeneracy (see also [13] where, however, the resulting expressions can still be simplified). Then there are eleven codim 2 bifurcations appearing as follows: (1) the critical normal form coefficients of a codim 1 bifurcation may vanish; (2) we can have strong resonances for the Neimark-Sacker bifurcation; or (3) more eigenvalues may arrive at the unit circle. In this paper we focus on those cases where the bifurcating fixed point has no more than two eigenvalues on the unit circle. The center manifold for such bifurcations is one- or two-dimensional. Normal forms for all of these cases have been derived long ago. Descriptions of the bifurcations involved can be found in $[1,3,2,14,17]$. However, except for the cusp $[13,14,5]$ and fold-flip [17], no explicit formulas for the critical coefficients in $n$-dimensional cases seem to be published. For plane diffeomorphisms, the advantage of our technique is that the linear part of the map need not be transformed into the Jordan form.

As a counterpart to diffeomorphisms, one may consider the center manifold reduction for codim 2 bifurcations of equilibria of vector fields. Explicit formulas for critical normal form coefficients at all five such codim 2 bifurcations have been derived in [15]. In the present paper we use a suitably adapted version of the same technique that combines the normalization with the center manifold reduction. This technique originated in [8].

The paper is organized as follows. In section 2 we review the codim 1 bifurcations and give a list of all generic codim 2 bifurcations of fixed points. In section 3 we discuss technicalities of the center manifold reduction for maps. In section 4 , which is the main part of the paper, we consider eight codim 2 bifurcations with at most two critical eigenvalues. For each of them, we give a critical normal form and derive its coefficients in the $n$-dimensional case. In section 5 we apply the developed techniques to two examples. First, we consider a three-dimensional map appearing in adaptive control. Using our analytical methods we are able to explain numerical observations reported in [9]. Then we study a periodically forced epidemic model and compute numerically the critical normal form coefficients for several codim 2 bifurcations occurring in this model. To compute the required derivatives of the Poincaré map, the automatic differentiation package ADOL-C is used.

2. Codim 1 and $\mathbf{2}$ bifurcations of fixed points. Write (1.1) at some parameter values as

$$
x \mapsto F(x), \quad F: \mathbb{R}^{n} \rightarrow \mathbb{R}^{n},
$$

and assume that it has a fixed point $x=0$. If the Jacobian matrix $A=F_{x}(0)$ has no eigenvalue $\lambda$ with $|\lambda|=1$, i.e., for a hyperbolic fixed point, the dynamics near the origin is topologically equivalent to that of the linear map $x \mapsto A x$ (GrobmanHartman theorem). If eigenvalues with $|\lambda|=1$ are present, the center manifold theorem guarantees the existence of stable, unstable, and center invariant manifolds 
near the fixed point. On the stable and unstable manifolds, the local dynamics is still determined by the linear part of the map. In contrast, the dynamics in the center manifold depends on both linear and nonlinear terms. Not all nonlinear terms are equally important, since some of them can be eliminated by an appropriate smooth coordinate transformation that puts the map restricted to the center manifold into a normal form, at least up to some order. Nonhyperbolic fixed points bifurcate; i.e., the dynamics near such points changes topologically under parameter variations. The birth of extra invariant objects, such as cycles or tori, depends on the critical normal form. Even though neither the center manifold nor the critical normal form on it are unique, the qualitative conclusions do not depend on the choices that are made.

Assuming sufficient smoothness of $F$, we write

$F(x)=A x+\frac{1}{2} B(x, x)+\frac{1}{6} C(x, x, x)+\frac{1}{24} D(x, x, x, x)+\frac{1}{120} E(x, x, x, x, x)+O\left(\|x\|^{6}\right)$,

where $A=F_{x}(0)$ and the components of the multilinear functions $B, C, D$, and $E$ are given by

$$
\begin{aligned}
B_{i}(x, y) & =\sum_{j, k=1}^{n} \frac{\partial^{2} F_{i}(0)}{\partial \xi_{j} \partial \xi_{k}} x_{j} y_{k} \\
C_{i}(x, y, z) & =\sum_{j, k, l=1}^{n} \frac{\partial^{3} F_{i}(0)}{\partial \xi_{j} \partial \xi_{k} \partial \xi_{l}} x_{j} y_{k} z_{l}, \\
D_{i}(x, y, z, u) & =\sum_{j, k, l, m=1}^{n} \frac{\partial^{4} F_{i}(0)}{\partial \xi_{j} \partial \xi_{k} \partial \xi_{l} \partial \xi_{m}} x_{j} y_{k} z_{l} u_{m}, \\
E_{i}(x, y, z, u, v) & =\sum_{j, k, l, m, s=1}^{n} \frac{\partial^{5} F_{i}(0)}{\partial \xi_{j} \partial \xi_{k} \partial \xi_{l} \partial \xi_{m} \partial \xi_{s}} x_{j} y_{k} z_{l} u_{m} v_{s}
\end{aligned}
$$

for $i=1,2, \ldots, n$. Here and in what follows $\|x\|=\sqrt{\langle x, x\rangle}$, where $\langle u, v\rangle=\bar{u}^{T} v$ is the standard scalar product in $\mathbb{C}^{n}\left(\right.$ or $\left.\mathbb{R}^{n}\right)$.

It is well known that there are three generic codim 1 bifurcations of fixed points. We first list the corresponding critical normal forms and their coefficients. These can be found, for example, in [14]; we will re-derive them in section 4 .

2.1. Fold. The matrix $A$ has a simple eigenvalue $\lambda_{1}=1$ and no other eigenvalues on the unit circle, while the restriction of (1.1) to a one-dimensional center manifold at the critical parameter value has the form

$$
w \mapsto w+\frac{1}{2} a w^{2}+O\left(w^{3}\right), \quad w \in \mathbb{R}^{1},
$$

where $a \neq 0$. When the control parameter crosses the critical value, two fixed points collide and disappear. For the coefficient $a$ we have the expression

$$
a=\langle p, B(q, q)\rangle,
$$

where $A q=q, A^{T} p=p$, and $\langle p, q\rangle=1$. 
2.2. Flip. The matrix $A$ has a simple eigenvalue $\lambda_{1}=-1$ and no other eigenvalues on the unit circle. The restriction of (1.1) to a one-dimensional center manifold at the critical parameter value can be transformed to the normal form

$$
w \mapsto-w+\frac{1}{6} b w^{3}+O\left(w^{4}\right), \quad w \in \mathbb{R}^{1},
$$

where $b \neq 0$. When the control parameter crosses the critical value, a cycle of period 2 bifurcates from the fixed point. This phenomenon is often called a period-doubling bifurcation. We have

$$
b=\left\langle p, C(q, q, q)+3 B\left(q,(I-A)^{-1} B(q, q)\right)\right\rangle,
$$

where $I$ is the unit $n \times n$ matrix, $A q=-q, A^{T} p=-p$, and $\langle p, q\rangle=1$.

2.3. Neimark-Sacker. The matrix $A$ has simple critical eigenvalues $\lambda_{1,2}=$ $e^{ \pm i \theta_{0}}$ and no other eigenvalues on the unit circle. Assume that

$$
e^{i k \theta_{0}} \neq 1, \quad k=1,2,3,4 \text { (no strong resonances). }
$$

Then, the restriction of (1.1) to a two-dimensional center manifold at the critical parameter value can be transformed to the normal form

$$
w \mapsto w e^{i \theta_{0}}\left(1+\frac{1}{2} d|w|^{2}\right)+O\left(|w|^{4}\right), \quad w \in \mathbb{C}^{1},
$$

where $w$ is now a complex variable and $d$ is a complex number. Further assume that the first Lyapunov coefficient

$$
c=\operatorname{Re} d \neq 0 \text {. }
$$

Under the above assumptions, a unique closed invariant curve around the fixed point appears when the parameter crosses the critical value. One has the following expression for $d$ :

$$
d=e^{-i \theta_{0}}\left\langle p, C(q, q, \bar{q})+2 B\left(q,(I-A)^{-1} B(q, \bar{q})\right)+B\left(\bar{q},\left(e^{2 i \theta_{0}} I-A\right)^{-1} B(q, q)\right)\right\rangle,
$$

where $A q=e^{i \theta_{0}} q, A^{T} p=e^{-i \theta_{0}} p$, and $\langle p, q\rangle=1$.

2.4. Codimension 2 cases. The following eleven codim 2 bifurcations can be met in generic two-parameter families of maps (1.1):

$$
\begin{array}{lll}
D_{1} & : & \lambda_{1}=1, a=0 \text { (cusp); } \\
D_{2} & : & \lambda_{1}=-1, b=0 \text { (generalized flip); } \\
D_{3} & : & \lambda_{1,2}=e^{ \pm i \theta_{0}}, c=0 \text { (Chenciner point); } \\
D_{4} & : & \lambda_{1}=\lambda_{2}=1(1: 1 \text { resonance); } \\
D_{5} & : & \lambda_{1}=\lambda_{2}=-1(1: 2 \text { resonance); } \\
D_{6} & : & \lambda_{1,2}=e^{ \pm i \theta_{0}}, \theta_{0}=\frac{2 \pi}{3}(1: 3 \text { resonance); } \\
D_{7} & : & \lambda_{1,2}=e^{ \pm i \theta_{0}}, \theta_{0}=\frac{\pi}{2}(1: 4 \text { resonance); } \\
D_{8} & : & \lambda_{1}=1, \lambda_{2}=-1 \text { (fold-flip); } \\
D_{9}: & \lambda_{1}=1, \lambda_{2,3}=e^{ \pm i \theta_{0}} \\
D_{10} & : & \lambda_{1}=-1, \lambda_{2,3}=e^{ \pm i \theta_{0}} \\
D_{11} & : & \lambda_{1,2}=e^{ \pm i \theta_{0}}, \lambda_{3,4}=e^{ \pm i \theta_{1}}
\end{array}
$$


At bifurcations $D_{1}$ and $D_{2}$ the matrix $A$ has one critical eigenvalue, while at $D_{3}{ }^{-}$ $D_{8}$ two critical eigenvalues are present. Cases $D_{9}$ and $D_{10}$ require three critical eigenvalues, while in the last case $D_{11}$ four critical eigenvalues must be present. In this paper we focus on the cases $D_{1}-D_{8}$ with most attention to $D_{2}-D_{7}$. The critical coefficients for the cusp case $\left(D_{1}\right)$ have been derived in $[13,14]$, while those for the fold-flip case $\left(D_{8}\right)$ can be found in [17]. Nevertheless, for completeness, we present formulas for the critical coefficients also in these two cases.

3. Center manifold reduction combined with normalization. Suppose that (2.1) has a nonhyperbolic fixed point $x=0$ and that the map obtained by restriction to the center manifold is transformed to a normal form

$$
w \mapsto G(w), \quad G: \mathbb{R}^{n_{c}} \rightarrow \mathbb{R}^{n_{c}},
$$

where $n_{c}$ is the number of the critical eigenvalues (counting multiplicity), or, equivalently, the dimension of the center manifold. Locally the critical center manifold can be parametrized by $w \in \mathbb{R}^{n_{c}}$ :

$$
x=H(w), \quad H: \mathbb{R}^{n_{c}} \rightarrow \mathbb{R}^{n} .
$$

Since the critical center manifold is invariant, we obtain the following homological equation for $H$ :

$$
H(G(w))=F(H(w))
$$

Our aim is to find the coefficients of $G$ at the bifurcation point. We approximate the center manifold using a power series in $w$. The coefficients of $G$ and $H$ can then be found by an iterative procedure.

We write Taylor series for $G$ and $H$

$$
G(w)=\sum_{|\nu| \geq 1} \frac{1}{\nu !} g_{\nu} w^{\nu}, \quad H(w)=\sum_{|\nu| \geq 1} \frac{1}{\nu !} h_{\nu} w^{\nu},
$$

where $g_{\nu}$ are normal form coefficients and $\nu=\left(\nu_{1}, \nu_{2}, \ldots, \nu_{n}\right)$ with $\nu !=\nu_{1} ! \nu_{2} ! \cdots \nu_{n}$ ! and $|\nu|=\nu_{1}+\nu_{2}+\cdots+\nu_{n}$. From the normal form theory, we know whether $g_{\nu}$ is nonzero but we do not know its value. If we expand (3.1) in powers of $w$, then we obtain a linear system for each $h_{\nu}$

$$
L h_{\nu}=R_{\nu}
$$

where $L$ is a matrix defined in terms of the Jacobian matrix $A$ and its critical eigenvalues. The right-hand side of (3.2) depends on the coefficients of $F, G$, and $H$ of order less than or equal to $|\nu|$. When $R_{\nu}$ involves only known quantities, the equation has a solution, because either $L$ is nonsingular or $R_{\nu}$ satisfies Fredholm's solvability condition

$$
\left\langle p, R_{\nu}\right\rangle=0
$$

where $p$ is any null-vector of the adjoint matrix $\bar{L}^{T}$. If $R_{\nu}$ depends on a critical coefficient $g_{\nu}$ of $G$, then $L$ is singular and the solvability condition gives the expression for $g_{\nu}$.

For codim 2 bifurcations we may need to find $h_{\nu}$, while $L$ is singular. Using the bordering technique we can solve such equations. Let $M$ be a complex singular $n \times n$ 
matrix with the one-dimensional null-space spanned by $q$, an eigenvector corresponding to a simple eigenvalue 0 of $M$. Similarly, let $p$ be an eigenvector corresponding to the eigenvalue 0 of the matrix $\bar{M}^{T}$. Then we construct the nonsingular $(n+1) \times(n+1)$ matrix

$$
\left(\begin{array}{cc}
M & q \\
\bar{p}^{T} & 0
\end{array}\right)
$$

Thus we are able to solve the system

$$
\left(\begin{array}{cc}
M & q \\
\bar{p}^{T} & 0
\end{array}\right)\left(\begin{array}{c}
x \\
s
\end{array}\right)=\left(\begin{array}{l}
y \\
0
\end{array}\right)
$$

where $s$ is a complex variable. We write $x=M^{I N V} y$. Since the vector on the righthand side is orthogonal to the critical eigenspace of the eigenvalue 0, i.e., $\langle p, y\rangle=0$, we will find that $s=0$. Note that the solution vector $x$ also satisfies $\langle p, x\rangle=0$; this property is used to simplify some expressions below.

4. Critical coefficients for codim 2 bifurcations. In this section we list critical normal forms on center manifolds for all codim 2 bifurcations with at most two critical eigenvalues as derived in [14]. Since higher-order terms do not affect subsequent computations, we give only truncated normal forms. Then we apply the center manifold reduction combined with normalization to derive explicit formulas for the critical normal form coefficients.

4.1. Cusp. In this case, there is only one critical eigenvalue +1 and the coefficient $a$ given by (2.2) vanishes. The truncated normal form for this bifurcation is

$$
w \mapsto w+\frac{1}{6} c w^{3},
$$

where $w \in \mathbb{R}^{1}$ is a local coordinate along the one-dimensional center manifold. We introduce two vectors such that

$$
A q=q, \quad A^{T} p=p .
$$

Note that we can choose the vectors $p$ and $q$ such that $\langle p, q\rangle=1$. Collecting the $w^{2}$ terms in (3.1) we find the equation

$$
(A-I) h_{2}=-B(q, q) .
$$

The matrix on the left-hand side is singular and the right-hand side satisfies Fredholm's solvability condition, since the defining condition for a cusp is $a=\langle p, B(q, q)\rangle=$ 0 , see (2.2). Therefore we use the bordering technique to solve for $h_{2}$. Next, we move on to the cubic terms and obtain

$$
(A-I) h_{3}=c q-C(q, q, q)-3 B\left(q, h_{2}\right) .
$$

This is another singular linear system. Using Fredholm's solvability condition and the normalization of $q$ with respect to $p$, we can express the critical coefficient $c$ as

$$
c=\left\langle p, C(q, q, q)-3 B\left(q,(A-I)^{I N V} B(q, q)\right)\right\rangle .
$$

The same formula is obtained in $[13,14]$. This case is also considered in [5], where the analysis is unnecessarily complicated. 
4.2. Generalized flip. In this case, there is only one critical eigenvalue -1 and the coefficient $b$ given by (2.3) vanishes. The truncated normal form is

$$
w \mapsto-w+\frac{1}{120} c w^{5},
$$

where $w \in \mathbb{R}^{1}$ is a properly selected local coordinate along the one-dimensional center manifold. We can find eigenvectors such that

$$
A q=-q, \quad A^{T} p=-p, \quad\langle p, q\rangle=1 .
$$

Collecting the $w^{2}$ terms in (3.1) we obtain

$$
(A-I) h_{2}=-B(q, q),
$$

which is a nonsingular linear system that has $h_{2}=(I-A)^{-1} B(q, q)$ as unique solution. We continue with the $w^{3}$ terms to get

$$
(A+I) h_{3}=-C(q, q, q)-3 B\left(q, h_{2}\right) .
$$

This singular system is solvable since

$$
\left\langle p, C(q, q, q)+3 B\left(q, h_{2}\right)\right\rangle=0
$$

according to (2.3). The fourth-order terms in (3.1) give

$$
(A-I) h_{4}=-\left(4 B\left(q, h_{3}\right)+3 B\left(h_{2}, h_{2}\right)+6 C\left(q, q, h_{2}\right)+D(q, q, q, q)\right) .
$$

This is a nonsingular system and thus we can solve for $h_{4}$. Finally, the critical coefficient $c$ appears in the fifth-order terms

$$
\begin{aligned}
(A+I) h_{5}=c q- & \left(5 B\left(q, h_{4}\right)+10 C\left(q, q, h_{3}\right)+10 B\left(h_{2}, h_{3}\right)\right. \\
+ & \left.15 C\left(q, h_{2}, h_{2}\right)+10 D\left(q, q, q, h_{2}\right)+E(q, q, q, q, q)\right) .
\end{aligned}
$$

The solvability of this singular linear system implies

$$
\begin{aligned}
c= & \left\langle p, 5 B\left(q, h_{4}\right)+10 C\left(q, q, h_{3}\right)+10 B\left(h_{2}, h_{3}\right)\right. \\
& \left.+15 C\left(q, h_{2}, h_{2}\right)+10 D\left(q, q, q, h_{2}\right)+E(q, q, q, q, q)\right\rangle .
\end{aligned}
$$

If $c \neq 0$, then the generalized flip is nondegenerate.

4.3. Chenciner bifurcation. This bifurcation occurs when there is a pair of complex eigenvalues with modulus one and the first Lyapunov coefficient vanishes. It is also assumed that there are no other critical eigenvalues. The truncated normal form is

$$
w \mapsto e^{i \theta_{0}} w+\frac{1}{2} c_{1} w|w|^{2}+\frac{1}{12} d_{1} w|w|^{4}, \quad e^{i k \theta_{0}} \neq 1 \quad \text { for } k=1,2,3,4,5,6 .
$$

Here $w \in \mathbb{C}^{1}$ is a suitable local complex coordinate on the center manifold. The first Lyapunov coefficient $l_{1}=\operatorname{Re}\left(e^{-i \theta_{0}} c_{1}\right)$ vanishes at the codim 2 point, while the value of its imaginary part may not vanish. We choose complex eigenvectors such that

$$
\begin{array}{r}
A q=e^{i \theta_{0}} q, \quad A \bar{q}=e^{-i \theta_{0}} \bar{q}, \\
A^{T} p=e^{-i \theta_{0}} p, \quad A^{T} \bar{p}=e^{i \theta_{0}} \bar{p} .
\end{array}
$$


These can be scaled such that $\langle p, q\rangle=1$. Collecting the $w^{j} \bar{w}^{k}$ terms in (3.1), we find the following equations to be satisfied:

quadratic $(j+k=2)$ :

$$
\begin{aligned}
\left(A-e^{2 i \theta_{0}} I\right) h_{20} & =-B(q, q), \\
(A-I) h_{11} & =-B(q, \bar{q}), \\
\left(A-e^{-2 i \theta_{0}} I\right) h_{02} & =-B(\bar{q}, \bar{q}),
\end{aligned}
$$

cubic $(j+k=3)$ :

$$
\begin{aligned}
\left(A-e^{3 i \theta_{0}} I\right) h_{30} & =-C(q, q, q)-3 B\left(q, h_{20}\right), \\
\left(A-e^{i \theta_{0}} I\right) h_{21} & =c_{1} q-C(q, q, \bar{q})-B\left(\bar{q}, h_{20}\right)-2 B\left(q, h_{11}\right), \\
\left(A-e^{-i \theta_{0}} I\right) h_{12} & =\overline{c_{1}} \bar{q}-C(q, \bar{q}, \bar{q})-B\left(q, h_{02}\right)-2 B\left(\bar{q}, h_{11}\right), \\
\left(A-e^{-3 i \theta_{0}} I\right) h_{03} & =-C(\bar{q}, \bar{q}, \bar{q})-3 B\left(\bar{q}, h_{02}\right) .
\end{aligned}
$$

As an intermediate result we find the expression leading to (2.4) for the coefficient $c_{1}$

$$
c_{1}=\left\langle p, C(q, q, \bar{q})-B\left(\bar{q},\left(A-e^{2 i \theta_{0}} I\right)^{-1} B(q, q)\right)-2 B\left(q,(A-I)^{-1} B(q, \bar{q})\right)\right\rangle .
$$

quartic $(j+k=4)$ :

$$
\begin{aligned}
\left(A-e^{4 i \theta_{0}} I\right) h_{40}=- & {\left[D(q, q, q, q)+6 C\left(q, q, h_{20}\right)+3 B\left(h_{20}, h_{20}\right)+4 B\left(q, h_{30}\right)\right], } \\
\left(A-e^{2 i \theta_{0}} I\right) h_{31}=- & {\left[D(q, q, q, \bar{q})+3 C\left(q, q, h_{11}\right)+3 C\left(q, \bar{q}, h_{20}\right)+3 B\left(q, h_{21}\right)\right.} \\
& \left.+3 B\left(h_{11}, h_{20}\right)+B\left(\bar{q}, h_{30}\right)\right]+3 c_{1} h_{20} e^{i \theta_{0}}, \\
(A-I) h_{22}=- & {\left[D(q, q, \bar{q}, \bar{q})+C\left(q, q, h_{02}\right)+C\left(\bar{q}, \bar{q}, h_{20}\right)+4 C\left(q, \bar{q}, h_{11}\right)\right.} \\
& \left.+B\left(h_{20}, h_{02}\right)+2 B\left(h_{11}, h_{11}\right)+2 B\left(q, h_{12}\right)+2 B\left(\bar{q}, h_{21}\right)\right] \\
+ & 2 h_{11}\left(c_{1} e^{-i \theta_{0}}+\bar{c}_{1} e^{i \theta_{0}}\right), \\
\left(A-e^{-2 i \theta_{0}} I\right) h_{13}=- & {\left[D(q, \bar{q}, \bar{q}, \bar{q})+3 C\left(\bar{q}, \bar{q}, h_{11}\right)+3 C\left(q, \bar{q}, h_{02}\right)+3 B\left(\bar{q}, h_{12}\right)\right.} \\
& \left.+3 B\left(h_{11}, h_{02}\right)+B\left(q, h_{03}\right)\right]+3 \bar{c}_{1} h_{02} e^{-i \theta_{0}}, \\
\left(A-e^{-4 i \theta_{0}} I\right) h_{04}=- & {\left[D(\bar{q}, \bar{q}, \bar{q}, \bar{q})+6 C\left(\bar{q}, \bar{q}, h_{02}\right)+3 B\left(h_{02}, h_{02}\right)+4 B\left(\bar{q}, h_{03}\right)\right], }
\end{aligned}
$$

quintic $(j+k=5)$ :

$$
\begin{aligned}
&\left(A-e^{i \theta_{0}} I\right) h_{32}=d_{1} q- {\left[E(q, q, q, \bar{q}, \bar{q})+D\left(q, q, q, h_{02}\right)+B\left(h_{02}, h_{30}\right)+6 B\left(h_{11}, h_{21}\right)\right.} \\
&+3 B\left(h_{20}, h_{12}\right)+6 C\left(q, h_{11}, h_{11}\right)+3 C\left(q, q, h_{12}\right)+6 C\left(q, \bar{q}, h_{21}\right) \\
&+6 C\left(\bar{q}, h_{11}, h_{20}\right)+C\left(\bar{q}, \bar{q}, h_{30}\right)+3 C\left(q, h_{20}, h_{02}\right)+2 B\left(\bar{q}, h_{31}\right) \\
&\left.+3 B\left(q, h_{22}\right)+6 D\left(q, q, \bar{q}, h_{11}\right)+3 D\left(q, \bar{q}, \bar{q}, h_{20}\right)\right] \\
&+6 h_{21}\left(c_{1}+\frac{1}{2} \bar{c}_{1} e^{2 i \theta_{0}}\right) .
\end{aligned}
$$

Having in mind implementation issues, we remark that computational time can be saved since $h_{21}=\bar{h}_{12}$ and $h_{20}=\bar{h}_{02}$. Also the vectors $h_{03}, h_{40}, h_{13}, h_{04}$, and $h_{32}$ need not be computed to find the critical normal form coefficient. Further, only for $h_{21}$ do we need to use the bordering technique. 
Finally, taking into account $\left\langle p, h_{21}\right\rangle=0$, we obtain the expression

$$
\begin{aligned}
d_{1}=\langle & p\left(q(q, q, q, \bar{q}, \bar{q})+D\left(q, q, q, h_{02}\right)+6 D\left(q, q, \bar{q}, h_{11}\right)+3 D\left(q, \bar{q}, \bar{q}, h_{20}\right)\right. \\
& +3 C\left(q, h_{20}, h_{02}\right)+6 C\left(q, h_{11}, h_{11}\right)+3 C\left(q, q, h_{12}\right)+6 C\left(q, \bar{q}, h_{21}\right) \\
& +6 C\left(\bar{q}, h_{11}, h_{20}\right)+C\left(\bar{q}, \bar{q}, h_{30}\right)+3 B\left(h_{20}, h_{12}\right)+6 B\left(h_{11}, h_{21}\right) \\
& \left.+3 B\left(q, h_{22}\right)+B\left(h_{02}, h_{30}\right)+2 B\left(\bar{q}, h_{31}\right)\right\rangle .
\end{aligned}
$$

The Chenciner bifurcation is nondegenerate if the second Lyapunov coefficient $l_{2}=$ $\operatorname{Re}\left(e^{-i \theta_{0}} d_{1}\right)+\frac{1}{2} \operatorname{Im}\left(e^{-i \theta_{0}} c_{1}\right)^{2}$ is nonzero.

4.4. Resonance 1:1. We have 1:1 resonance if there are two eigenvalues equal to 1 and no other critical eigenvalues exist. The truncated normal form for this bifurcation is

$$
\left(\begin{array}{c}
w_{1} \\
w_{2}
\end{array}\right) \mapsto\left(\begin{array}{c}
w_{1}+w_{2} \\
w_{2}+\frac{1}{2} a w_{1}^{2}+b w_{1} w_{2}
\end{array}\right),
$$

where $w \in \mathbb{R}^{2}$ provides a suitable local parametrization of the two-dimensional center manifold. We can find (generalized) eigenvectors of $A$ such that

$$
A q_{0}=q_{0}, \quad A q_{1}=q_{1}+q_{0}
$$

and similarly for the transposed matrix $A^{T}$

$$
A^{T} p_{0}=p_{0}, \quad A^{T} p_{1}=p_{1}+p_{0},
$$

so that $\left\langle p_{0}, q_{1}\right\rangle=\left\langle p_{1}, q_{0}\right\rangle=1,\left\langle p_{0}, q_{0}\right\rangle=\left\langle p_{1}, q_{1}\right\rangle=0$.

Collecting the $w^{2}$ terms in (3.1), we obtain the singular linear systems

$$
\begin{aligned}
& w_{1}^{2}:(A-I) h_{20}=-B\left(q_{0}, q_{0}\right)+a q_{1}, \\
& w_{1} w_{2}:(A-I) h_{11}=-B\left(q_{0}, q_{1}\right)+h_{20}+b q_{1}, \\
& w_{2}^{2}:(A-I) h_{02}=-B\left(q_{1}, q_{1}\right)+2 h_{11}+h_{20} .
\end{aligned}
$$

The solvability of these systems requires that their right-hand sides should be orthogonal to $p_{0}$. This gives

$$
a=\left\langle p_{0}, B\left(q_{0}, q_{0}\right)\right\rangle, \quad b=\left\langle p_{0}, B\left(q_{0}, q_{1}\right)\right\rangle+\left\langle p_{1}, B\left(q_{0}, q_{0}\right)\right\rangle .
$$

There is a Neimark-Sacker bifurcation curve emerging from the codim 2 point. If $s=(b-a) a<0$, the bifurcating invariant curve will be stable.

4.5. Resonance 1:2. Here we have two eigenvalues equal to -1 and no other critical eigenvalues. The truncated normal form is

$$
\left(\begin{array}{c}
w_{1} \\
w_{2}
\end{array}\right) \mapsto\left(\begin{array}{c}
-w_{1}+w_{2} \\
-w_{2}+\frac{1}{6} c w_{1}^{3}+\frac{1}{2} d w_{1}^{2} w_{2}
\end{array}\right),
$$

where $w \in \mathbb{R}^{2}$ is a suitable local parametrization of the critical center manifold. First we find generalized eigenvectors of $A$ and $A^{T}$

$$
\begin{aligned}
A q_{0} & =-q_{0}, & A q_{1}=-q_{1}+q_{0}, \\
A^{T} p_{0} & =-p_{0}, & A^{T} p_{1}=-p_{1}+p_{0},
\end{aligned}
$$


satisfying the same normalization conditions as at 1:1 resonance. Collecting the quadratic terms in (3.1) we get

$$
\begin{aligned}
& w_{1}^{2}:(A-I) h_{20}=-B\left(q_{0}, q_{0}\right), \\
& w_{1} w_{2}:(A-I) h_{11}=-B\left(q_{0}, q_{1}\right)-h_{20}, \\
& w_{2}^{2}:(A-I) h_{02}=-B\left(q_{1}, q_{1}\right)-2 h_{11}+h_{20} .
\end{aligned}
$$

Notice that the matrix $(A-I)$ is nonsingular, since $A$ has only a double eigenvalue $\lambda=-1$ on the unit circle. Therefore, one can solve these equations for $h_{20}, h_{11}$, and $h_{02}$ in the usual way.

From the cubic terms we will find the equations

$$
\begin{aligned}
w_{1}^{3}: & (A+I) h_{30}=c q_{1}-3 B\left(q_{0}, h_{20}\right)-C\left(q_{0}, q_{0}, q_{0}\right), \\
w_{1}^{2} w_{2} & :(A+I) h_{21}=d q_{1}+h_{30}-2 B\left(q_{0}, h_{11}\right)-B\left(q_{1}, h_{20}\right)-C\left(q_{0}, q_{0}, q_{1}\right), \\
w_{1} w_{2}^{2}:(A+I) h_{12} & =2 h_{21}-h_{30}-2 B\left(q_{1}, h_{11}\right)-B\left(q_{0}, h_{02}\right)-C\left(q_{0}, q_{1}, q_{1}\right), \\
w_{2}^{3}:(A+I) h_{03} & =3\left(h_{12}-h_{21}\right)+h_{30}-3 B\left(q_{1}, h_{02}\right)-C\left(q_{1}, q_{1}, q_{1}\right) .
\end{aligned}
$$

Now we can easily determine the critical coefficient $c$

$$
c=\left\langle p_{0}, C\left(q_{0}, q_{0}, q_{0}\right)+3 B\left(q_{0},(I-A)^{-1} B\left(q_{0}, q_{0}\right)\right)\right\rangle .
$$

The equation for the critical coefficient $d$ involves the vector $h_{30}$. Taking the scalar product with $p_{1}$, we find that $\left\langle p_{0}, h_{30}\right\rangle=-\left\langle p_{1}, 3 B\left(q_{0}, h_{20}\right)+C\left(q_{0}, q_{0}, q_{0}\right)\right\rangle$ from the equation at the $w_{1}^{3}$ term. Then the solvability of the equation for $h_{21}$ implies

$$
d=\left\langle p_{0}, 2 B\left(q_{0}, h_{11}\right)+B\left(q_{1}, h_{20}\right)+C\left(q_{0}, q_{0}, q_{1}\right)\right\rangle+\left\langle p_{1}, 3 B\left(q_{0}, h_{20}\right)+C\left(q_{0}, q_{0}, q_{0}\right)\right\rangle .
$$

Note that only the quadratic approximation of the center manifold is computed explicitly. The nondegeneracy conditions are $C_{1}=2 c / 3 \neq 0$ and $D_{1}=-d-c \neq 0$. If $C_{1}<0$, then the critical point is a saddle, if $C_{1}>0$, then the critical point is elliptic. The coefficient $D_{1}$ determines the bifurcation scenario under generic perturbations (see [14] for details).

4.6. Resonance 1:3. Similar to the Chenciner bifurcation, the normal form for the resonance 1:3 has been studied in the complex form. However, one quadratic term in the normal form cannot be eliminated due to the appearance of two complex conjugated eigenvalues which are cubic roots of unity. The truncated normal form is given by

$$
w \mapsto e^{i \theta_{0}} w+\frac{1}{2} b \bar{w}^{2}+\frac{1}{2} c w|w|^{2}, \quad \theta_{0}=\frac{2 \pi}{3},
$$

where $w \in \mathbb{C}^{1}$ is a normalizing local coordinate on the center manifold. Now we select complex eigenvectors such that

$$
\begin{gathered}
A q=e^{i \theta_{0}} q, \quad A \bar{q}=e^{-i \theta_{0}} \bar{q}, \\
A^{T} p=e^{-i \theta_{0}} p, \quad A^{T} \bar{p}=e^{i \theta_{0}} \bar{p},
\end{gathered}
$$

and $\langle p, q\rangle=1$. The quadratic part of the homological equation (3.1) gives

$$
\begin{aligned}
w^{2} & :\left(A-e^{2 i \theta_{0}} I\right) h_{20}=\bar{b} \bar{q}-B(q, q), \\
w \bar{w} & :(A-I) h_{11}=-B(q, \bar{q}), \\
\bar{w}^{2} & :\left(A-e^{-2 i \theta_{0}} I\right) h_{02}=b q-B(\bar{q}, \bar{q}) .
\end{aligned}
$$


We remark that the first and third equations are complex conjugated, so $\bar{h}_{20}=h_{02}$. Second, since $e^{i 2 \pi / 3}$ is an eigenvalue of $A$, we have a singularity implying that $h_{02}$ should be found using a bordered system. As before, the solvability condition gives

$$
b=\langle p, B(\bar{q}, \bar{q})\rangle .
$$

We only collect the $z^{2} \bar{z}$ terms, since this is all we need to find the critical coefficient $c$,

$$
\left(A-e^{i \theta_{0}} I\right) h_{21}=c q+e^{-i \theta_{0}} \bar{b} h_{02}-2 B\left(q, h_{11}\right)-B\left(\bar{q}, h_{20}\right)-C(q, q, \bar{q}) .
$$

From (4.14) it follows that $\left\langle p, h_{02}\right\rangle=0$, so that we obtain the expression for $c$, which is similar to that of the Neimark-Sacker coefficient if $b=0$, cf. (2.4),

$$
c=\left\langle p, C(q, q, \bar{q})+2 B\left(q,(I-A)^{-1} B(q, \bar{q})\right)-B\left(\bar{q},\left(e^{2 i \theta_{0}} I-A\right)^{I N V}(\bar{b} \bar{q}-B(q, q))\right)\right\rangle .
$$

If $b \neq 0$, the real part of $c_{1}=\frac{3}{4}\left(2 e^{i 4 \pi / 3} c-|b|^{2}\right)$ determines the stability of the bifurcating invariant closed curve.

4.7. Resonance 1:4. For this bifurcation the quadratic terms can be eliminated, but two cubic terms cannot be neglected. The truncated normal form is given using a normalizing complex coordinate $w \in \mathbb{C}^{1}$ on the center manifold by

$$
w \mapsto e^{i \theta_{0}} w+\frac{1}{2} c w|w|^{2}+\frac{1}{6} d \bar{w}^{3}, \quad \theta_{0}=\frac{\pi}{2} .
$$

As usual, we select complex eigenvectors such that

$$
A q=e^{i \theta_{0}} q, \quad A \bar{q}=e^{-i \theta_{0}} \bar{q}, \quad A^{T} p=e^{-i \theta_{0}} p, \quad A^{T} \bar{p}=e^{i \theta_{0}} \bar{p}, \quad\langle p, q\rangle=1 .
$$

The quadratic part of (3.1) gives

$$
\begin{aligned}
w^{2}:(A+I) h_{20} & =-B(q, q), \\
w \bar{w}:(A-I) h_{11} & =-B(q, \bar{q}), \\
\bar{w}^{2}:(A+I) h_{02} & =-B(\bar{q}, \bar{q}) .
\end{aligned}
$$

Since \pm 1 are not the eigenvalues of $A$, we can easily find $h_{20}, h_{11}$, and $h_{02}$. Now as above we only collect the coefficient in front of the resonant terms:

$$
\begin{aligned}
& \left(A-e^{i \theta_{0}} I\right) h_{21}=c q-2 B\left(q, h_{11}\right)-B\left(\bar{q}, h_{20}\right)-C(q, q, \bar{q}), \\
& \left(A-e^{i \theta_{0}} I\right) h_{03}=d q-3 B\left(\bar{q}, h_{02}\right)-C(\bar{q}, \bar{q}, \bar{q}) .
\end{aligned}
$$

The solvability conditions imply

$$
c=\left\langle p, C(q, q, \bar{q})+2 B\left(q,(I-A)^{-1} B(q, \bar{q})\right)-B\left(\bar{q},(I+A)^{-1} B(q, q)\right)\right\rangle
$$

and

$$
d=\left\langle p, C(\bar{q}, \bar{q}, \bar{q})-3 B\left(\bar{q},(I+A)^{-1} B(\bar{q}, \bar{q})\right)\right\rangle
$$

If $d \neq 0$, then

$$
A_{0}=-\frac{3 i c}{|d|}
$$

determines the bifurcation scenario near the 1:4 point (see details in [14]). 
4.8. Fold-flip. For completeness, we include here the results from [17], where the formulas for center manifold reduction for the fold-flip bifurcation have been derived. This bifurcation is characterized by two simple eigenvalues on the unit circle, one +1 and one -1 . The hypernormal form is

$$
\left(\begin{array}{l}
x \\
y
\end{array}\right) \mapsto\left(\begin{array}{l}
x+\frac{1}{2} a_{0} x^{2}+\frac{1}{2} b_{0} y^{2}+\frac{1}{6} c_{0} x^{3}+\frac{1}{2} d_{0} x y^{2} \\
-y+x y
\end{array}\right) .
$$

The matrix $A$ has the eigenvalues $\lambda_{1,2}= \pm 1$. Introduce the associated eigenvectors $q_{1}, q_{2}, p_{1}, p_{2}$, such that

$$
\begin{array}{ccc}
A q_{1}=q_{1}, & A^{T} p_{1}=p_{1}, & \left\langle p_{1}, q_{1}\right\rangle=1 \\
A q_{2}=-q_{2}, & A^{T} p_{2}=-p_{2}, & \left\langle p_{2}, q_{2}\right\rangle=1 .
\end{array}
$$

First one computes the coefficients $a_{1}, b_{1}, e_{1}$

$$
a_{1}=\left\langle p_{1}, B\left(q_{1}, q_{1}\right)\right\rangle, \quad e_{1}=\left\langle p_{2}, B\left(q_{2}, q_{1}\right)\right\rangle, \quad b_{1}=\left\langle p_{1}, B\left(q_{2}, q_{2}\right)\right\rangle .
$$

Then we have to compute the quadratic approximation of the center manifold. As before we use the bordering technique to find

$$
\begin{aligned}
& h_{20}=(A-I)^{I N V}\left(\left\langle p_{1}, B\left(q_{1}, q_{1}\right)\right\rangle q_{1}-B\left(q_{1}, q_{1}\right)\right), \\
& h_{11}=(A+I)^{I N V}\left(\left\langle p_{2}, B\left(q_{1}, q_{2}\right)\right\rangle q_{2}-B\left(q_{1}, q_{2}\right)\right), \\
& h_{02}=(A-I)^{I N V}\left(\left\langle p_{1}, B\left(q_{2}, q_{2}\right)\right\rangle q_{1}-B\left(q_{2}, q_{2}\right)\right) .
\end{aligned}
$$

Then as another intermediate result we find the coefficients $c_{i}$

$$
\begin{aligned}
& c_{1}=\left\langle q_{1}, C\left(q_{1}, q_{1}, q_{1}\right)+3 B\left(q_{1}, h_{20}\right)\right\rangle, \\
& c_{2}=\left\langle q_{1}, C\left(q_{1}, q_{2}, q_{2}\right)+B\left(q_{1}, h_{02}\right)+2 B\left(q_{2}, h_{11}\right)\right\rangle, \\
& c_{3}=\left\langle p_{2}, C\left(q_{1}, q_{1}, q_{2}\right)+B\left(q_{2}, h_{20}\right)+2 B\left(q_{1}, h_{11}\right)\right\rangle, \\
& c_{4}=\left\langle p_{2}, C\left(q_{2}, q_{2}, q_{2}\right)+3 B\left(q_{2}, h_{02}\right)\right\rangle .
\end{aligned}
$$

Provided that $e_{1} \neq 0$, the coefficients for (4.19) are given by

$$
a_{0}=\frac{a_{1}}{e_{1}}, \quad b_{0}=b_{1} e_{1}, \quad c_{0}=\frac{c_{1}}{e_{1}^{2}}, \quad d_{0}=c_{2}+\frac{1}{e_{1}}\left(b_{1} c_{3}-\frac{1}{3}\left(2 e_{1}+a_{1}\right) c_{4}\right) .
$$

The nondegeneracy conditions are $a_{0}, b_{0} \neq 0$. If $a_{0}<0$ and $b_{0}>0$, then also $3 a_{0} b_{0}+a_{0} d_{0}+a_{0}^{2} b_{0}-b_{0} c_{0} \neq 0$ is required.

5. Examples. In this section we apply the results obtained in the previous section to two examples. The first is a map in $\mathbb{R}^{3}$ from the theory of adaptive control [9]. The second example is a seasonally forced epidemic model [18], defined by a system of time-periodic ODEs in $\mathbb{R}^{3}$, for which the Poincaré map and its derivatives have to be computed numerically. In both examples, we study codim 2 bifurcations of fixed points.

5.1. Adaptive control map. Consider a model for controlling a single-input/ single-output plant. The process to be controlled is discrete and the control law is linear. Golden and Ydstie [10] have introduced a specific example where the output 
is regulated to a constant by a low-order feedback. This leads to the following map $G: \mathbb{R}^{3} \mapsto \mathbb{R}^{3}$ :

$$
G:\left(\begin{array}{c}
x \\
y \\
z
\end{array}\right) \mapsto\left(\begin{array}{c}
y \\
b x+k+z y \\
z-\frac{k y}{c+y^{2}}(b x+k+z y-1)
\end{array}\right)
$$

The coefficient $b$ measures the mismatch between the reference and the real model. The coefficient $k$ represents an error in the assumption on how strong the output variable is controlled. Choosing $b=0, k=1$ implies no errors. The coefficient $c$ comes from the controller design. It is positive to avoid division by zero. For a control mechanism it is best to have as little complicating behavior as possible. Since the possible bifurcation sequences are determined by the critical normal form coefficients, we may choose the constant $c$ to prevent too complex behavior. The system was studied in [9] numerically near strong resonance points. A derivation of the map can also be found in this paper.

There are two codim 1 bifurcation curves for fixed points with several codim 2 points. We can compute the second- and third-order derivatives of this map analytically. This allows us to compute the normal form coefficients for the strong resonances analytically and to study their dependence on $c$.

This map has one fixed point given by $(x, y, z)=(1,1,1-b-k)$. Local bifurcation analysis reveals two codim 1 bifurcation curves shown in Figure 5.1. For

$$
b_{f}=1-\left(\frac{1}{2}+\frac{1}{4(c+1)}\right) k
$$

the fixed point undergoes a period-doubling, while if

$$
b_{N S}=-\left(\frac{c+1}{c+2}\right)
$$

and $k^{2}+4 b k<0$, an invariant curve emerges from the fixed point via the NeimarkSacker bifurcation.

Starting from $k=0$ on the Neimark-Sacker curve and tracing it until we meet the period-doubling curve, we encounter strong resonances. These occur for the following values of $k$ :

$$
(1: 4) \quad k=\frac{2(c+1)}{c+2}, \quad(1: 3) \quad k=\frac{3(c+1)}{c+2}, \quad(1: 2) \quad k=\frac{4(c+1)}{c+2} .
$$

As is observed in [9], at $(k, c)=(1.308 \ldots, 0.1)$ the Neimark-Sacker bifurcation is degenerate.

In Appendix A we provide the expressions for the relevant eigenvectors and the derivatives of (5.1) up to and including the fifth-order.

- Resonance 1:2. A straightforward computation using (4.11), (4.12), and the expressions from Appendix A yields

$$
\begin{aligned}
& C_{1}=\frac{4(2+c)^{2}\left(1-3 c-2 c^{2}-2 c^{3}\right)}{(3+2 c)(1+c)^{2}}, \\
& D_{1}=\frac{(2+c)^{2}\left(-25+51 c+40 c^{2}+56 c^{3}+8 c^{4}\right)}{(3+2 c)(1+c)^{2}} .
\end{aligned}
$$

For small $c$ up to $c \approx 0.347$ the coefficient $D_{1}$ is negative, while $C_{1}$ is positive up to $c \approx 0.271$. 


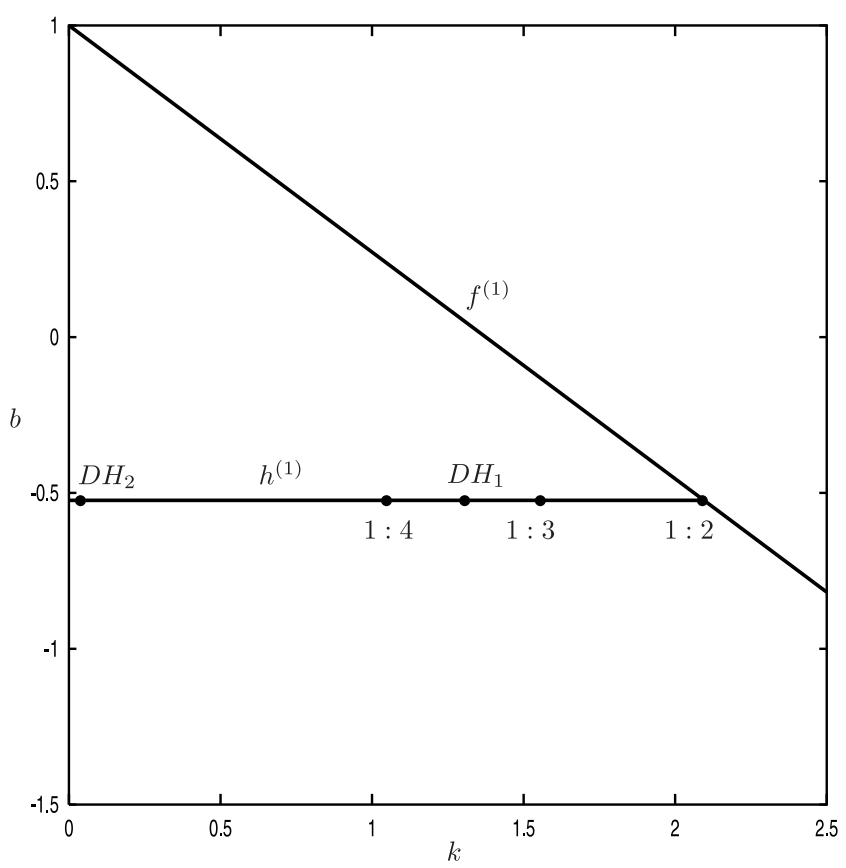

FIG. 5.1. Local bifurcations of (5.1) for $c=1 / 10: f^{(1)}-$ flip (period-doubling), $h^{(1)}-$ NeimarkSacker bifurcation of the fixed point. Labels $(1: k)$ denote the strong resonances; $D H_{1,2}$ are the Chenciner points. The codim 2 points are studied in this paper.

- Resonance 1:3. Now we compute $c$ by formula (4.15) and find

$$
\operatorname{Re}_{1}=\frac{3(2+c)\left(-22-105 c-76 c^{2}+155 c^{3}+237 c^{4}+111 c^{5}+18 c^{6}\right)}{2(1+c)^{2}\left(7+9 c+3 c^{2}\right)^{2}} .
$$

Since $c>0$ but small, this means that the invariant closed curve existing close to the $1: 3$ bifurcation is stable.

- Resonance 1:4. This bifurcation has the most complicated bifurcation sequences. Using (4.17) and (4.18), we get

$$
A_{0}(c)=\frac{\left(2+c-4 c^{2}+3 c^{3}+2 c^{4}\right)-i\left(1+36 c+40 c^{2}+17 c^{3}+4 c^{4}\right)}{\sqrt{\left(5+6 c+2 c^{2}\right)\left(1-10 c+83 c^{2}+12 c^{3}+4 c^{4}+8 c^{5}+2 c^{6}\right)}} \operatorname{sgn}(3+2 c) .
$$

Consider now the case with small positive $c$. The real part of $A_{0}$ is always positive. If we start with $c=0$ we find $\left|A_{0}\right|=1$, and thus we will encounter the bifurcation sequences IV(a), III(a), III, V, V(a), VI, and VIII (here the labeling from [14] is used). Actually, for small negative $c$ we are within the unit circle in the $A_{0}$-plane, and the dynamics is simple.

- Chenciner bifurcation. For the Neimark-Sacker bifurcation we derive using (2.4) the following expression for the first Lyapunov coefficient:

$$
\begin{aligned}
l_{1}= & \left(b(1+b)^{2}(1+2 b)\left(4+4 b+3 b^{2}\right)+\left(4+21 b+50 b^{2}+102 b^{3}+92 b^{4}-7 b^{5}\right) k\right. \\
& \left.+\left(14 b+72 b^{2}+79 b^{3}-3 b^{4}\right) k^{2}+(1+3 b)(3+5 b) k^{3}\right) \\
& /\left(2 b^{2}(1+b)\left((1+b)^{2}+k\right)\left(b(1+b)^{2}+4 b k+k^{2}\right)\right) .
\end{aligned}
$$


For $c=1 / 10$ we have $b_{N S}=-11 / 21$ and $l_{1}$ vanishes at two points, namely, $D H_{1}$ with $k \approx 1.30064$ and $D H_{2}$ with $k \approx 0.0214$ (see Figure 5.1). The first point has been reported in [9] but not analyzed; the second point was overlooked.

For $c=0.1, b \approx-.52381$, and $k \approx 1.30064$, we find $e^{-i \theta} c_{1} \approx 0.754395 i$. As we have already mentioned, the first Lyapunov coefficient vanishes close to this point. We compute the derivatives up to fifth order (see Appendix A) and then solve recursively for $h_{j k}$. This gives

$$
\begin{aligned}
& h_{20} \approx(-1.4588-1.5723 i, 0.5516+2.0727 i, 1.6970-1.7342 i), \\
& h_{11} \approx(-0.7019,-0.7019,1.1871), \\
& h_{30} \approx(-4.7567-2.9281 i,-5.3569+1.5823 i, 4.2621-4.3465 i), \\
& h_{21} \approx(1.5723-1.7334 i, 1.9215+1.1523 i, 3.1354-2.1245 i), \\
& h_{31} \approx(-6.4614+18.7153 i, 9.7894-12.2548 i, 1.9848-3.2357 i), \\
& h_{22} \approx(5.6473-12.5220 i, 5.6473-12.5220 i, 21.4770-5.9938 i) .
\end{aligned}
$$

Using these values for the second Lyapunov coefficient (4.7) we find $d_{1} \approx-28.546$. The same procedure can be carried out for the second point yielding $d_{1} \approx 26720.8$. This implies that both Chenciner bifurcations in (5.1) are nondegenerate.

From a control point of view, coexistence of the fixed point together with stable attractors is undesirable. In the original paper [9] numerical continuation of fixed points and computation of one-dimensional stable and unstable manifolds were used. The coexistence of global stable attractors together with the period-one fixed point and tangencies of stable and unstable manifolds were then deduced from the phase portraits. The authors pointed out that it was difficult to characterize the bifurcations near the codim 2 points. However, first, we computed the critical normal form coefficients symbolically and thus we were able to check that these are indeed nondegenerate codim 2 bifurcations. Second, we were able to verify the hypothesis that some global bifurcations occurred. Since these are present in the normal form for certain values of the critical normal form coefficients, by continuity they should also be observed away from the resonances. The analysis showed that a specific choice of $c>0$ may lead to more desirable bifurcation sequences.

5.2. Example: SEIR-model. The SEIR epidemic model describes the spread of a nonlethal disease in a large population, which is divided into four classes: susceptible $(S)$, exposed $(E)$, infective $(I)$, and recovered $(R)$. Let us briefly introduce the model (see [6] for details and references). New susceptibles are "born" with the growth rate $\mu ; \beta$ is the contact rate between susceptibles and infectives. The exposed become infective with the rate $\alpha$ and the infectives recover with the rate $\gamma$. This gives

$$
\left\{\begin{array}{c}
\dot{S}=\mu-\mu S-\beta S I, \\
\dot{E}=\beta S I-(\mu+\alpha) E, \\
\dot{I}=\alpha E-(\mu+\gamma) I,
\end{array}\right.
$$

and $R=1-S-E-I$. In [18] effects of a seasonal variation of the contact rate with other parameters constant,

$$
\beta=\beta_{0}(1+\delta \cos (2 \pi t)),
$$

were studied numerically by considering the Poincaré map

$$
P:(S(0), E(0), I(0)) \mapsto(S(1), E(1), I(1)) .
$$




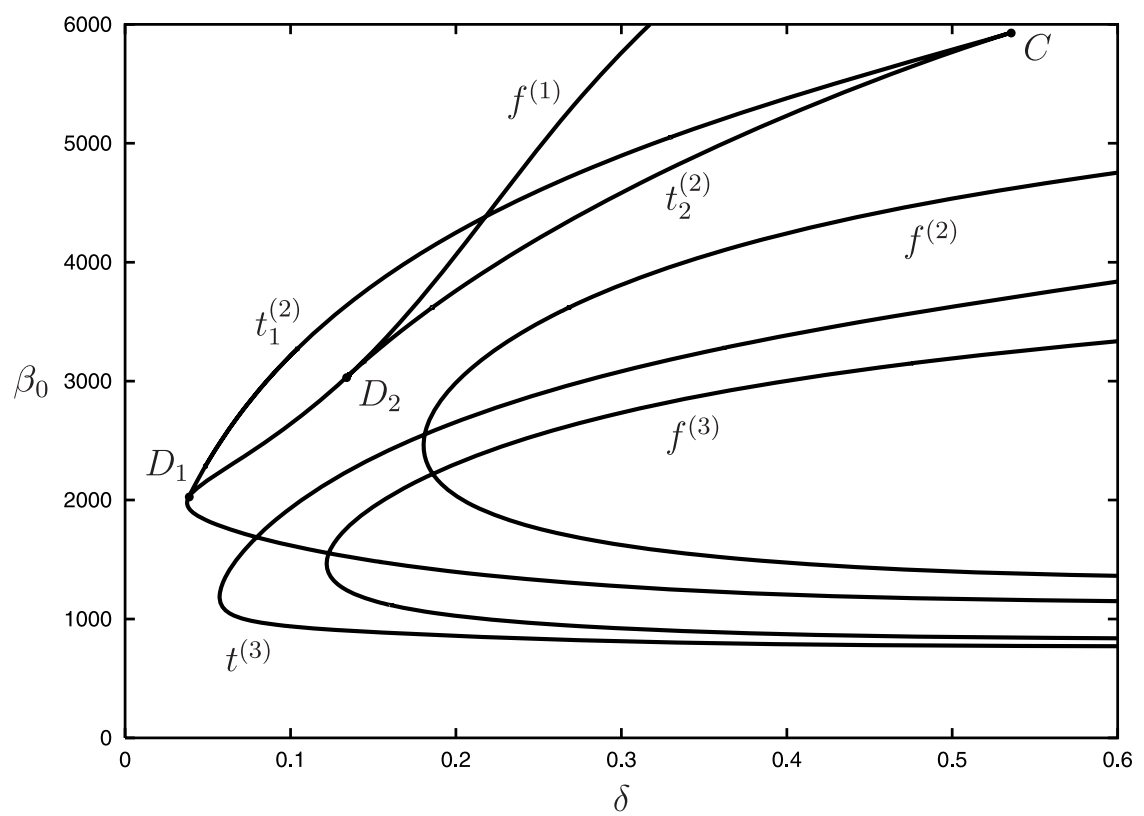

FIG. 5.2. Bifurcation diagram of the SEIR-model. Labels $t^{(k)}\left(f^{(k)}\right)$ denote fold (flip) bifurcation of period-k cycles. Point $C$ corresponds to the cusp bifurcation of period-2 cycles; $D_{1,2}$ are the degenerate flip points. The codim 2 points are studied in this paper.

For measles the characteristic parameters are $\mu=0.02, \alpha=35.842$, and $\gamma=100$. A bifurcation diagram in two parameters $(\beta$, the mean value, and $\delta$, the degree of seasonality) was obtained. Codim 2 bifurcations, namely, cusps and degenerate flips, were found.

We used CONTENT [16] to recompute the codim 1 bifurcation curves obtained in [18] and to locate the codim 2 points (see Figure 5.2):

\begin{tabular}{|l|l|l|}
\hline Point & Parameter & Coordinates \\
\hline$C$ & $\delta \approx 0.5327$ & $S \approx 0.02229$ \\
& $\beta_{0} \approx 5928$ & $E \approx 0.1887 \times 10^{-7}$ \\
& & $I \approx 0.5499 \times 10^{-8}$ \\
\hline$D_{1}$ & $\delta \approx 0.03815$ & $S \approx 0.05029$ \\
& $\beta_{0} \approx 2015$ & $E \approx 5.3301 \times 10^{-4}$ \\
& & $I \approx 1.8846 \times 10^{-4}$ \\
\hline$D_{2}$ & $\delta \approx 0.1328$ & $S \approx 0.03566$ \\
& $\beta_{0} \approx 3019$ & $E \approx 4.9463 \times 10^{-4}$ \\
& & $I \approx 1.6794 \times 10^{-4}$ \\
\hline
\end{tabular}

The Poincaré map (5.5) was computed using the Runge-Kutta-Fehlberg integration method of order 7-8 with the absolute step tolerance $10^{-14}$. The derivatives of the Poincaré map were obtained with the same accuracy as the Poincaré map using the automatic differentiation package ADOL-C [11] (see Appendix B). Then the formulas for the critical normal form coefficients derived in section 4 were implemented in MATLAB.

Instead of the original SEIR-model (5.4), we used an equivalent system for $s=$ $\log S, e=\log E$, and $i=\log I$. When the variables are small, integrating this equivalent system is numerically more stable. 
For the critical normal form coefficients (2.2) and (4.2) we have found

$$
\text { Cusp : } a \approx-7.53 \times 10^{-7}, \quad c \approx-0.224 \text {. }
$$

We see that the cusp point is indeed nondegenerate, so that precisely three cycles of period 2 exist for nearby parameter values.

For the degenerate flip points we have obtained using (2.3) and (4.4) the following values:

$$
\begin{aligned}
& D_{1}: b \approx-2.33 \times 10^{-6}, \quad c \approx 0.764, \\
& D_{2}: b \approx-1.49 \times 10^{-5}, \quad c \approx-0.0313 .
\end{aligned}
$$

Thus, these points are nondegenerate and one fold bifurcation curve $t_{k}^{(2)}$ emanates tangentially to $f^{(1)}$ from each codim 2 point $D_{k}$ for $k=1,2$ (see Figure 5.2).

Now consider the region enclosed by $f^{(1)}, t_{1}^{(2)}$, and $t_{2}^{(2)}$. Here two different stable period-two attractors coexist, one with two-yearly outbreaks, the other with yearly outbreaks. One comes from the curve $t_{1}^{(2)}$ originating in $D_{1}$. The other is a result of the period doubling when crossing $f^{(1)}$. This region exists, because the coefficients $c$ of $D_{1}$ and $D_{2}$ have opposing signs.

Since we verified the nondegeneracy numerically, this implies that we indeed deal with codim 2 points. So near the codim 2 points $D_{1,2}$ and $C$ no other bifurcations exist, and the picture is complete.

6. Discussion. In this paper we have derived the explicit formulas for the normal form coefficients to verify the nondegeneracy of codim 2 bifurcations of fixed points with one or two critical eigenvalues. In many previous studies, the nondegeneracy of such points was merely assumed. Applying our results to $n$-dimensional maps, one avoids the computation of the center manifold, but can directly evaluate the critical normal form coefficients in the original basis. Note that the formulas remain valid also for $n=2$ and allow one to avoid the transformation of the linear part of the map into Jordan form. Thus they can also be useful in the analysis of planar maps.

The derived formulas are suitable for a direct implementation in any symbolic manipulation or numerical package supporting complex arithmetic. We plan to implement these formulas for the Map class of dynamical systems in the next version of CONTENT [16].

Normal form coefficients for the remaining three codim 2 bifurcations of fixed points (cases $D_{9}, D_{10}$, and $D_{11}$ from section 2 ) can be easily obtained using the same normalization techniques. These formulas will be reported elsewhere, together with the detailed bifurcation analysis of the corresponding unfoldings.

Analysis of codim 2 bifurcations of limit cycles is a more delicate problem. The derived formulas above for the normal form coefficients can be applied also in this case, provided that all necessary derivatives of the Poincaré map are computed at its fixed point corresponding to the limit cycle. For nonstiff ODEs, this can be achieved either by integrating appropriate variational equations or using automatic differentiation. This gives the full derivatives of the $T$-shift along the orbits of the system, which should then be projected to the Poincaré cross section (see [17] for the first-, second-, and third-order derivatives). For stiff ODEs, this approach will not work, since even accurate computation of the Poincaré map may be problematic. In such cases, numerical techniques based on discretized boundary-value problems (BVPs) with the piecewise polynomial approximation of solutions and the orthogonal collocation proved to be suitable for the continuation of limit cycles and their bifurcations 
[7]. Development of robust numerical methods for the normal form analysis of limit cycle bifurcations which fit into the BVP framework remains an important research area.

Finally, let us mention that the full verification of the genericity of a codim 2 bifurcation would require not only establishing its nondegeneracy at the critical parameter values but also a careful analysis of how the system depends on parameters. Here we have not aimed at verifying the transversality of the bifurcations with respect to parameters. Nevertheless, the normalization technique used in the paper allows for a generalization to parameter-dependent maps, so that canonical unfolding parameters could be expressed in terms of the actual system parameters (see [17] for the fold-flip case and [4, Section 11], where some of the codim 2 cases for vector fields have been treated). In practice, a codim 2 singularity is often detected while computing codim 1 bifurcation curves. In such cases, the transversality with respect to parameters may be deduced from the tangency or transversality of these curves in the parameter plane at the codim 2 point.

Appendix A. Here we give the expressions of the eigenvectors on the NeimarkSacker curve along which all four codim 2 bifurcations occur. We fix $c=-\left(\frac{2 b+1}{b+1}\right)$ rather than $b$, since this simplifies formulas. The equivalent expressions can easily be computed.

The Jacobi matrix at the fixed point is

$$
A=\left(\begin{array}{ccc}
0 & 1 & 0 \\
b & 1-b-k & 1 \\
k(1+b) & \frac{k(1+b)(1-b-k)}{b} & 1+k+\frac{k}{b}
\end{array}\right)
$$

with eigenvalues

$$
\lambda_{1}=\frac{2 b+k-\sqrt{k(4 b+k)}}{2 b}, \quad \lambda_{2}=\frac{2 b+k+\sqrt{k(4 b+k)}}{2 b}, \quad \lambda_{3}=-b .
$$

If $k^{2}+4 b k<0$, then the eigenvectors corresponding to $\lambda_{1}$ are

$$
\begin{aligned}
& q=\left(-\frac{k(3 b+k)+(b+k) \sqrt{k(4 b+k)}}{2 k b(b+1)}, \frac{-k+\sqrt{k(4 b+k)}}{2 k(b+1)}, 1\right)^{T} \\
& p=\frac{1}{\alpha}\left(-\frac{1}{2}(k+\sqrt{k(4 b+k)}),-\frac{1}{2 b}(k(2 b+1)+\sqrt{k(4 b+k)}), 1\right)^{T},
\end{aligned}
$$

where

$$
\alpha=\frac{(1+b+k)(k+4 b)-(1+3 b+k) \sqrt{k^{2}+4 b}}{2 b(1+b)} .
$$

Then the derivatives are given by

$$
B(p, q)=\left(\begin{array}{c}
0 \\
p_{2} q_{3}+p_{3} q_{2} \\
\frac{(1+b)(2+3 b) k}{k}\left(p_{1} q_{2}+p_{2} q_{1}\right)+\frac{2(1+b)(2+3 b) k(1-b-k)}{b^{2}} p_{2} q_{2} \\
+\frac{(1+b)(1+2 b) k}{b^{2}}\left(p_{2} q_{3}+p_{3} q_{2}\right)
\end{array}\right) .
$$


It is easy to see that for the third- and higher-order tensors only the third component will be nonzero. We write

$$
C(p, q, r)=\left(\begin{array}{c}
0 \\
0 \\
\sum_{i, j, k=1}^{3} C_{i j k} p_{i} q_{j} r_{k}
\end{array}\right)
$$

where at least two indices of $C_{i j k}$ are equal to two, otherwise the coefficient is zero. We have

$$
\begin{gathered}
C_{122}=C_{212}=C_{221}=\frac{2(1+b)^{2}(4+7 b) k}{b^{2}}, \\
C_{222}=\frac{6(1+b)^{2}(4+7 b) k(1-b-k)}{b^{3}}, \\
C_{322}=C_{232}=C_{223}=\frac{2(1+b)^{2}(1+2 b)(4+5 b) k}{b^{3}} .
\end{gathered}
$$

In a similar manner we find

$$
D(p, q, r, s)=\left(\begin{array}{c}
0 \\
0 \\
\sum_{i, j, k, l=1}^{3} D_{i j k l} p_{i} q_{j} r_{k} s_{l}
\end{array}\right)
$$

where at least three indices of $D_{i j k l}$ are equal to two, otherwise the coefficient is zero. We have

$$
\begin{gathered}
D_{1222}=D_{2122}=D_{2212}=D_{2221}=\frac{6(1+b)^{2}\left(8+24 b+17 b^{2}\right) k}{b^{3}}, \\
D_{2222}=\frac{24(1+b)^{2}\left(8+24 b+17 b^{2}\right) k(1-b-k)}{b^{4}}, \\
D_{3222}=D_{2322}=D_{2232}=D_{2223}=\frac{24(1+b)^{2}(1+2 b)(2+3 b) k}{b^{4}} .
\end{gathered}
$$

And finally the fifth-order tensors are given by

$$
E(p, q, r, s, t)=\left(\begin{array}{c}
0 \\
0 \\
\sum_{i, j, k, l, m=1}^{3} E_{i j k l m} p_{i} q_{j} r_{k} s_{l} t_{m}
\end{array}\right),
$$

where

$$
\begin{aligned}
& E_{12222}=E_{21222}=E_{22122}=E_{22212}=E_{22221}=\frac{24(1+b)^{3}\left(16+52 b+41 b^{2}\right) k}{b^{4}}, \\
& E_{22222}=\frac{120(1+b)^{3}\left(16+52 b+41 b^{2}\right) k(1-b-k)}{b^{5}} \\
& E_{32222}=E_{23222}=E_{22322}=E_{22232}=E_{22223}=\frac{24(1+b)^{2}(1+2 b)\left(16+44 b+29 b^{2}\right) k}{b^{5}} \\
& \text { and all other coefficients are zero. }
\end{aligned}
$$


Appendix B. In section 5.2 we have studied codim 2 bifurcations of periodic solutions in the epidemic model. For the analysis we needed third- or even fifth-order derivatives of the Poincaré map. These numerical data can be obtained using ADOL-C [11], a package for automatic differentiation, that is more accurate and efficient than finite differences or integrating variational equations. Here we describe how we have used this package and give an example code.

We start with a solution $Y=Y(t)$ of a $T$-periodic ODE

$$
\dot{Y}=f(Y, t), \quad Y \in \mathbb{R}^{n} .
$$

A periodic solution corresponds to a fixed point of the Poincaré (period) map $Y(0) \mapsto$ $Y(T)=P(Y(0))$. Continuation of codim 1 bifurcations of fixed points can be done, for instance, in CONTENT [16]. Continuation using ADOL-C has been explored in [12]. Thus we may encounter a codim 2 bifurcation. To perform the normal form analysis of the codim 2 bifurcation, we need the derivatives of the Poincare map. For most cases, third-order derivatives are sufficient, but for some cases fifth-order tensors are required. These may be programmed as follows.

First the variables and parameters are initialized and then the active section is marked with the command trace_on. The initial point $Y(0)$ is passed by overloading to a variable which is of type adouble defined by ADOL-C. Then we integrate the system until time $T$ and get the final point $Y(T)$. This point is passed on to a passive variable, again by overloading, and we mark the end of the active section with the command trace_off. Now the derivatives can be obtained with the command tensor_eval and stored for the computation of the critical normal form coefficients. ${ }^{1}$ The following code in $\mathrm{C}++$ shows how the third-order derivatives for the cusp of section 5.2 can be computed.

Note that here we treat the periodic case only. For autonomous systems, the total derivative of the flow can be computed with ADOL-C and then projected on a Poincaré cross section, see [17].

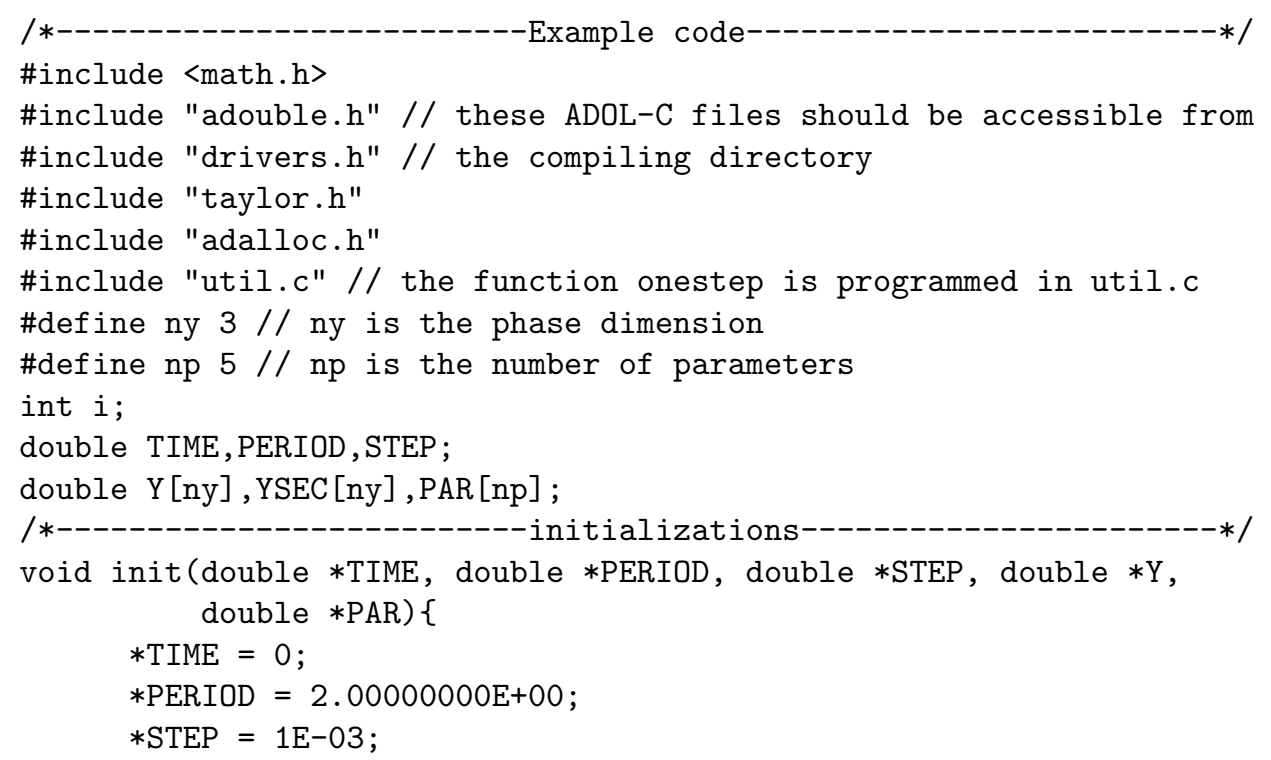

${ }^{1}$ We did this computation in MATLAB. 


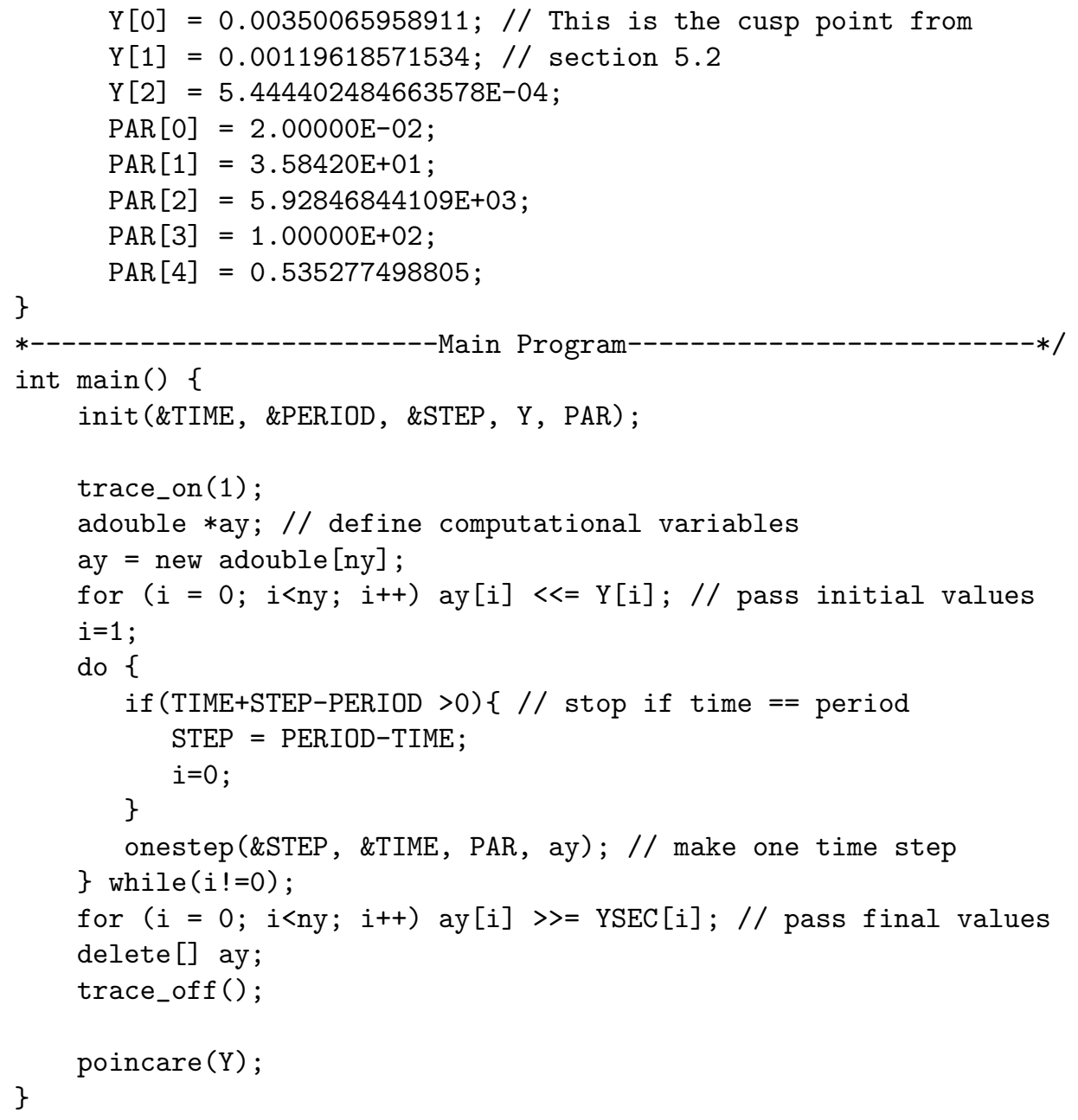

The function onestep should be written such that the adouble ay is passed to another adouble. In our code we assumed that a one-step integration method is used. This can be easily adjusted to multistep methods. Note also that the stepsize STEP is assumed to be automatically adjusted in onestep according to predefined tolerances.

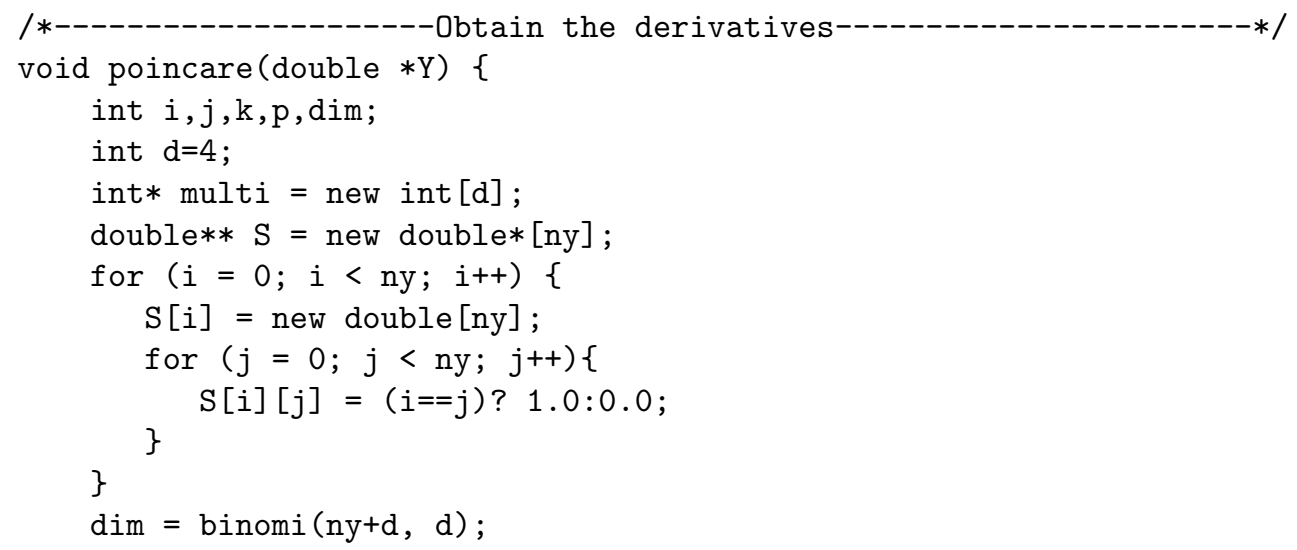




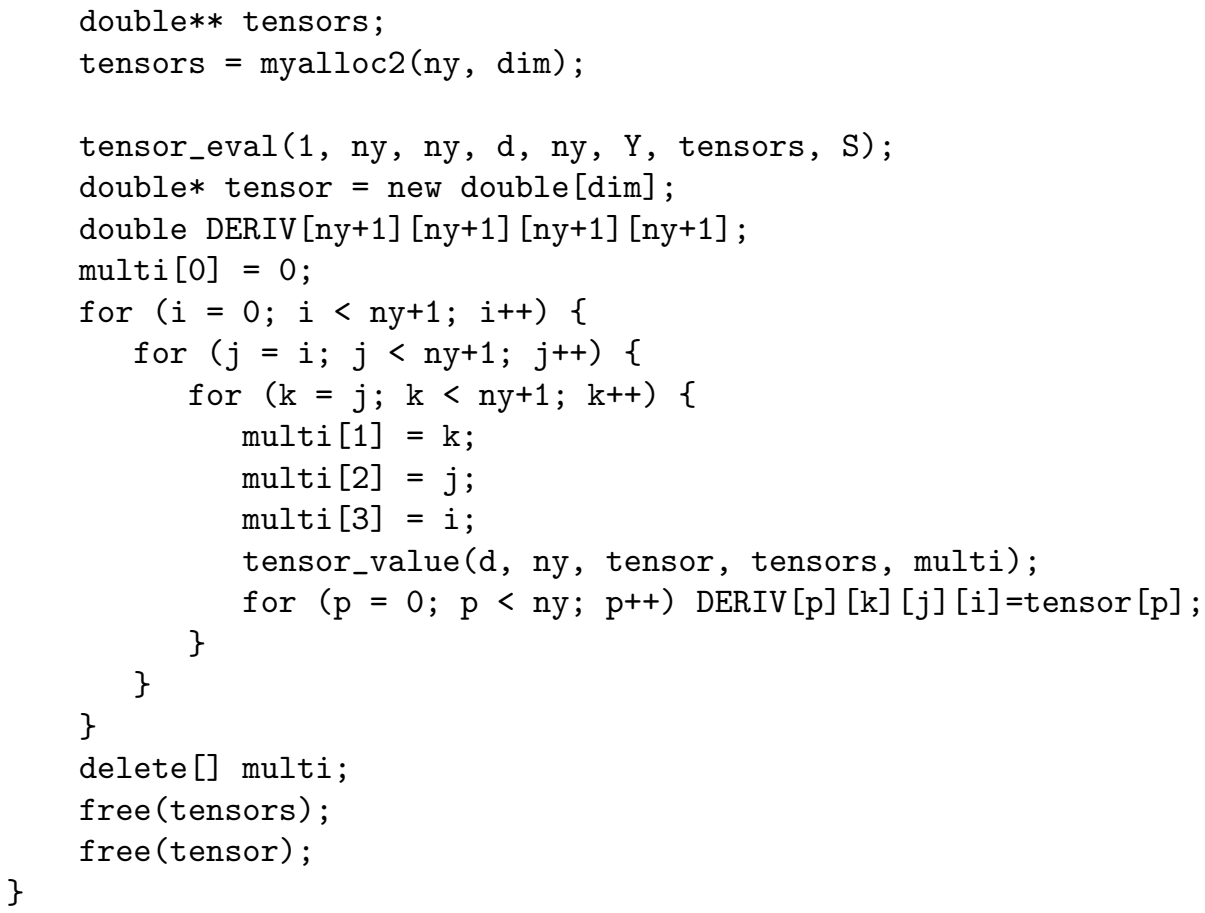

The variable DERIV will contain the required information as follows:

$$
\begin{array}{ll}
\operatorname{DERIV}[\mathrm{p}][\mathrm{i}][0][0]=\frac{\partial P_{p}(Y(0))}{\partial Y_{i}}, & i \geq 1, \\
\operatorname{DERIV}[\mathrm{p}][\mathrm{i}][j][0]=\frac{\partial^{2} P_{p}(Y(0))}{\partial Y_{i} \partial Y_{j}}, & i \geq j \geq 1, \\
\operatorname{DERIV}[\mathrm{p}][\mathrm{i}][j][\mathrm{k}]=\frac{\partial^{3} P_{p}(Y(0))}{\partial Y_{i} \partial Y_{j} \partial Y_{k}}, & i \geq j \geq k \geq 1,
\end{array}
$$

where the indices $i, j, k$ are nonincreasing and $p$ denotes the component of $P$. Thus, only one half of the derivatives is stored, which causes no problems if the map $P$ is smooth enough. These derivatives may be stored in a file for further analysis in MATLAB.

\section{REFERENCES}

[1] V.I. ARnold, Geometrical Methods in the Theory of Ordinary Differential Equations, SpringerVerlag, New York, 1983.

[2] V.I. Arnol'D, V.S. Afraimovich, Yu.S. Il'yashenko, and L.P. Shil'nikov, Bifurcation theory, in Dynamical Systems V, Encyclopaedia Math. Sci. 5, V.I. Arnold, ed., SpringerVerlag, New York, 1994, pp. 1-205.

[3] D. Arrowsmith and C. Place, An Introduction to Dynamical Systems, Cambridge University Press, Cambridge, 1990.

[4] W.-J. Beyn, A. Champneys, E. Doedel, W. Govearts, Yu.A. Kuznetsov, and B. SandstEDE, Numerical continuation, and computation of normal forms, in Handbook of Dynamical Systems, Vol. 2, B. Fiedler, ed., Elsevier Science, Amsterdam, 2002, pp. 149-219.

[5] J. CARCASSÈS AND H. KaWAKAmI, Existence of a cusp point on a fold bifurcation curve and stability of the associated fixed point. Case of an n-dimensional map, Internat. J. Bifur. Chaos Appl. Sci. Engrg., 5 (1999), pp. 875-894. 
[6] O. Diekmann and J. Heesterbeek, Mathematical Epidemiology of Infectious Diseases, John Wiley \& Sons, Ltd., Chichester, England, 2000.

[7] E. Doedel, H. Keller, And J. Kernévez, Numerical analysis and control of bifurcation problems: (II) Bifurcation in infinite dimensions, Internat. J. Bifur. Chaos Appl. Sci. Engrg., 1 (1991), pp. 745-772.

[8] C. Elphick, E. Tirapegui, M. Brachet, P. Coullet, and G. Iooss, A simple global characterization for normal forms of singular vector fields, Phys. D, 32 (1987), pp. 95-127.

[9] C. Frouzakis, R. Adomatis, and I. Kevrekidis, Resonance phenomena in an adaptivelycontrolled system, Internat. J. Bifur. Chaos Appl. Sci. Engrg., 1 (1991), pp. 83-106.

[10] M. Golden And B. Ydstie, Bifurcation in model reference adaptive control systems, Systems Control Lett., 11 (1988), pp. 413-430.

[11] A. Griewank, D. Juedes, H. Mitev, J. Utke, O. Vogel, and A. Walther, Adol-C: $a$ package for the automatic differentiation of algorithms written in $C / C++, \mathrm{ACM}$ Trans. Math. Software, 22 (1996), pp. 131-167.

[12] J. GuCKenheimer And B. Meloon, Computing periodic orbits and their bifurcations with automatic differentiation, SIAM J. Sci. Comput., 22 (2000), pp. 951-985.

[13] L.G. Kurakin, Semi-invariant form of criteria for stability of fixed points of a mapping in critical cases, Izv. Severo-Kavkaz. Nauchn. Tsentra Vyssh. Shkoly Estestv. Nauk., 141 (1989), pp. 31-35 (in Russian).

[14] Yu.A. Kuznetsov, Elements of Applied Bifurcation Theory, Springer-Verlag, Berlin, 1998.

[15] Yu.A. KuzNETSOv, Numerical normalization techniques for all codim 2 bifurcations of equilibria in ODE's, SIAM J. Numer. Anal., 36 (1999), pp. 1104-1124.

[16] Yu.A. Kuznetsov and V.V. Levitin, Content: A Multiplatform Environment for Analyzing Dynamical Systems, 1997. Available via ftp from ftp.cwi.nl/pub/CONTENT.

[17] Yu.A. Kuznetsov, H. Meijer, and L. van Veen, The fold-flip bifurcation, Internat. J. Bifur. Chaos Appl. Sci. Engrg., 7 (2004), pp. 2253-2282.

[18] Yu.A. Kuznetsov And C. PicCARdi, Bifurcation analysis of periodic SEIR and SIR epidemic models, J. Math. Biol., 32 (1994), pp. 109-121. 